\title{
DISTRIBUTION FUNCTIONS CONTAINING A GAUSSIAN FACTOR
}

\author{
HARRY POLLARD ${ }^{1}$
}

1. Introduction. In the present paper we shall obtain necessary and sufficient conditions that a distribution function $F(t)$ contain the normal distribution

$$
G(t)=\frac{1}{\pi^{1 / 2}} \int_{-\infty}^{t} e^{-u^{2}} d u
$$

as a factor in the sense that ${ }^{2}$

$$
F(x)=\int G(x-y) d \alpha(y), \quad-\infty<x<\infty,
$$

where $\alpha(y)$ is also a distribution function. If we denote the derivative of $F(x)$ by $f(x)$, the problem is clearly that of representing $f(x)$ in the form

$$
f(x)=\frac{1}{\pi^{1 / 2}} \int e^{-(x-y)^{2}} d \alpha(y), \quad-\infty<x<\infty .
$$

Several writers (for example see [1] and [4]) have obtained results from which criteria for our problem follow, but the conditions involve the analytic continuation of $f(x)$, or are otherwise cumbersome. We give a new solution as follows.

THEOREM. In order that a function $f(x)$ defined on $(-\infty, \infty)$ have the form (1.2), where $\alpha(y)$ is a distribution function, the following conditions are necessary and sufficient:

(i) $\int f(x) d x=1$;

(ii) $f(x) \in C^{\infty}$ on $(-\infty<x<\infty)$;

(iii) the series

$$
\sum(-)^{k} f^{(2 k)}(x) \frac{t^{k}}{4^{k} k !}
$$

converges to a non-negative value uniformly on $-\infty<x<\infty$ for each value of $t, 0 \leqq t<1$.

Received by the editors November 14, 1952.

${ }^{1}$ Research supported in part by a grant from the Office of Naval Research.

2 All integrals which occur henceforth are doubly infinite, and their limits are omitted. Similarly in all sums the limits are to be taken from 0 to $\infty$.

3 This can be replaced by uniformity in finite intervals without altering the proofs. It would be most desirable to drop the requirement of uniformity altogether. 
As an immediate consequence we have the following corollary:

COROLLARY. In order that the distribution function $F(t)$ have $G(t)$ as a factor it is necessary and sufficient that it be absolutely continuous and that its derivative $f(t)$ have properties (ii) and (iii).

Our method also leads to the following explicit determination of $\alpha(x)$ in terms of $F(x)$ :

$$
\text { (1.3) } \frac{1}{2}[\alpha(x+0)+\alpha(x-0)]=\lim _{t \rightarrow 1} \sum(-)^{k} F^{(2 k)}(x) \frac{t^{k}}{4^{k} k !} .
$$

A very interesting account of the developments which suggest conditions of the form (iii) is given by Widder [4].

2. The necessity. Suppose that $f(x)$ has the form (1.2). Then it obviously has property (i). That it has property (ii) follows from the fact that $e^{x^{2}} f(x)$ is a bilateral Laplace transform converging for $-\infty<x<\infty$. In fact $f(x)$ is entire and we may differentiate under the integral any number of times [4]. Therefore

$$
f^{(2 k)}(x)=\frac{1}{\pi^{1 / 2}} \int H_{2 k}(x-y) e^{-(x-y)^{2}} d \alpha(y),
$$

where $H_{n}(x)$ is the $n$th Hermite polynomial, defined by

$$
H_{n}(x)=(-)^{n} e^{x^{2}}\left(\frac{d}{d x}\right)^{n}\left(e^{-x^{2}}\right) .
$$

Now form the formal series

$$
\begin{aligned}
\sum(-1)^{k} f^{(2 k)}(x) \frac{t^{k}}{4^{k} k !} & \\
= & \frac{1}{\pi^{1 / 2}} \int \sum(-1)^{k} H_{2 k}(x-y) \frac{t^{k}}{4^{k} k !} e^{-(x-y)^{2}} d \alpha(y) .
\end{aligned}
$$

This step is justified if we establish the finiteness of

$$
\int \sum\left|H_{2 k}(x-y)\right| \frac{t^{k}}{4^{k} k !} e^{-(x-y)^{2}} d \alpha(y),
$$

a fact which follows directly from the inequality $[2$, p. 236]

$$
\left|H_{2 k}(x)\right| \leqq A 2^{k}[(2 k) !]^{1 / 2} e^{x^{2} / 2} .
$$

We now make use of the following identity $[2$, p. 371]: 


$$
\sum \frac{H_{n}(x)}{m !}\left(\frac{w}{2}\right)^{n}=\left(1+w^{2}\right)^{3 / 2}\left(1+x w+w^{2}\right) \exp \left(\frac{x^{2} w^{2}}{1+w^{2}}\right)
$$

where $m=[n / 2]$. Replace $w$ by $-w$, add the resulting equation to this one, and replace $w$ by $i t^{1 / 2}$. In this fashion we obtain the identity

$$
\sum(-1)^{n} \frac{H_{2 n}(x)}{n !} \frac{t^{n}}{4^{n}}=(1-t)^{-1 / 2} \exp \left(-\frac{x^{2} t}{1-t}\right)
$$

It follows that the right-hand member of (2.2) is

$$
\frac{1}{(\pi \tau)^{1 / 2}} \int e^{-(x-y)^{2} / \tau} d \alpha(y)
$$

where $\tau=1-t$; and this is non-negative, since $d \alpha \geqq 0$. This establishes the necessity of (iii) except for the assertion about uniformity. To fill in this lacuna apply (2.3) directly to (2.2). We get

$$
\begin{aligned}
\left|f^{(2 k)}(x)\right| & \leqq B 2^{k}[(2 k) !]^{1 / 2} \int e^{-(x-y)^{2} / 2} d \alpha(y) \\
& \leqq B 2^{k}[(2 k) !]^{1 / 2}
\end{aligned}
$$

since $\int d \alpha=1$. The series occurring in (iii) is then dominated by

$$
\sum[(2 k) !]^{1 / 2} \frac{t^{k}}{2^{k} k !}
$$

which converges when $0 \leqq t<1$.

3. The sufficiency. Define the function $u(x, \tau)$ by

$$
u(x, \tau)=\sum(-1)^{k} f^{(2 k)}(x) \frac{(1-\tau)^{k}}{4^{k} k !} .
$$

According to (iii) the convergence of this series is assured for $0<\tau \leqq 1$. Now let $\tau_{0}$ be fixed. In view of the assumption of uniformity in $x$ it follows that

$$
\left|f^{(2 k)}(x)\right| \frac{\left(1-\tau_{0}\right)^{k}}{4^{k} k !}<1
$$

for $k \geqq k\left(\tau_{0}\right)$. Now form the series

$$
\sum\left|f^{(2 k+2)}(x)\right| \frac{(1-\tau)^{k}}{4^{k} k !} .
$$

According to (3.2), if we choose $0<\tau_{0}<\tau$ the new series is dominated 
(from some term on) by

$$
\frac{1}{1-\tau_{0}} \sum \frac{4^{k+1}(k+1) !}{4^{k} k !}\left(\frac{1-\tau}{1-\tau_{0}}\right)^{k} \text {. }
$$

This establishes the uniform convergence of (3.3) as a series of functions of $x$.

As a consequence we may differentiate the series in (3.1) the requisite number of times to obtain the heat equation

$$
\frac{\partial^{2} u}{\partial x^{2}}=\frac{1}{4} \frac{\partial u}{\partial \tau}, \quad-\infty<x<\infty, 0 \leqq \tau<1 .
$$

Now, by condition (iii) the function $u(x, \tau)$ is non-negative. Therefore, by an important theorem of Widder [5], $u(x, \tau)$ must take the form ${ }^{4}$

$$
u(x, \tau)=\frac{1}{(\pi \tau)^{1 / 2}} \int e^{-(x-y)^{2} / \tau} d \alpha(y),
$$

where $\alpha(y)$ is an increasing function. According to (3.1) we have $\lim _{\tau \rightarrow 1} u(x, \tau)=f(x)$. Hence

$$
f(x)=\lim _{\tau \rightarrow 1} \frac{1}{\pi^{1 / 2}} \int e^{-(x-y)^{2} / \tau} d \alpha(y) .
$$

Since $\alpha$ as a set function defines a completely additive measure, we may invoke Fatou's lemma to assure us that this last integral converges at $\tau=1$. Therefore by Lebesgue's principle of dominated convergence we may take the limit under the integral to obtain (1.2).

It remains only to show that $\int d \alpha=1$. But, according to (1.2), $\int f(x) d x=\int d \alpha$, and by (i) this has the value 1 .

4. The inversion formula. Suppose now that $F(x)$ does have the representation (1.1). Then

$$
F(x)=\int \alpha(x-y) d G(y)=\frac{1}{\pi^{1 / 2}} \int e^{-(x-y)^{2}} \alpha(y) d y .
$$

Note that $\alpha(y)$ is a bounded function. This justifies our repeating the steps leading from (2.2) to (2.4) to derive the formula

$$
\sum(-1)^{k} F^{(2 k)}(x) \frac{t^{k}}{k ! 4^{k}}=\frac{1}{(\pi \tau)^{1 / 2}} \int e^{-(x-y)^{2} / \tau} \alpha(y) d y,
$$

4 This device is used by Widder in his paper [4], but we have completed the argument in a different way. 
where $1-t=\tau$. It is known that the right-hand member approaches $[\alpha(x+0)+\alpha(x-0)] / 2$ as $\tau \rightarrow 0$ (see $[3$, p. 31]) and this establishes (1.3).

\section{REFERENCES}

1. H. Pollard, Representation as a Gaussian integral, Duke Math. J. vol. 10 (1943) pp. 59-65.

2. G. Szeg8, Orthogonal polymomials, New York, 1939.

3. E. C. Titchmarsh, Fourier integrals, Oxford, 1937.

4. D. V. Widder, Necessary and sufficient conditions for the representation of a function by a Weierstrass transform, Trans. Amer. Math. Soc. vol. 71 (1951) pp. 430-439. 5. - Positive temperatures on an infinite rod, Trans. Amer. Math. Soc. vol. 55 (1944) pp. 85-95.

The Institute for Advanced Study 